\title{
The Integrative Medicine in China
}

\author{
Wei Zheng ${ }^{\mathrm{a}}$ \\ Henan Academy Institute of Traditional Chinese \\ Medicine, Zhengzhou, Henan Province, 450000, China \\ E-mail: questwz@163.com
}

\author{
Zhang Junping ${ }^{\mathrm{c}}$ \\ Henan Academy Institute of Traditional Chinese \\ Medicine, Zhengzhou, Henan Province, 450000, China \\ E-mail: zhjunpingi0372@163.com
}

\author{
Zhao faxin ${ }^{\mathrm{b}}$ \\ Henan Academy Institute of Traditional Chinese \\ Medicine, Zhengzhou, Henan Province, 450000, China \\ E-mail: hnzhaofaxin@163.com \\ Zhao Guocen ${ }^{\mathrm{d}^{*}}$ \\ Henan Academy Institute of Traditional Chinese \\ Medicine, Zhengzhou, Henan Province, 450000, China \\ E-mail: 277789196@qq.com
}

\begin{abstract}
This paper describes the historical evolution of the concept of the combination of traditional Chinese medicine (TCM) and Western medicine and notes that the combination of the two is a knowledge system based on their common development, not simply the westernization of TCM. Both of them have their advantages and should learn from each other. A combination method is further proposed: Combining TCM and Western medicine from the clinical perspective; Combining TCM and Western medicine in terms of experimental studies; the use of modern technology to achieve the modernization of TCM. Ways to promote the internationalization of the combination are also pointed out: establishing an international multi-center observation for clinical trials to TCM; Emphasizing the quality of papers of TCM and publishing more SCI papers to conduct discussion. This paper concludes that we must take full advantages of TCM, work out our way, and develop independent property rights to enhance national self-confidence. By learning from Western medicine, we enhance TCM. The old saying "learn from the advanced technologies in the West in order to resist the invasion of the Western powers" still has significant meaning in the field of combining the TCM and Western medicine.
\end{abstract}

Keywords-The Combination of TCM and Western medicine; Theoretical Exploration; Methodology

\section{THE DEFINITION OF THE COMBINATION OF TCM AND WESTERN MEDICINE}

In 1956, Chairman Mao Zedong proposed to combine TCM and western medicine and establish unified medicine and pharmacy system in China. Since then, the concept of the combination has been formed gradually. Personally, the combination of TCM and western medicine is a knowledge system based on both, rather than the simple westernization of TCM. TCM and western medicine are different, for they have different diagnostic criteria and methods; however, they are all used to serve patients and save their lives. Therefore, it is necessary for them to learn from others' strong point, offset their own weakness, and avoid mutual attack. Some treatment in western medicine, like assay and imaging, could function as a supplement of the four ways of diagnosis "look, listen, question and feel the pulse" of TCM, while its "differential treatment" could also enlighten the "evidence-based medicine" and "individual treatment" of western medicine. They are gradually combined in the constant collision. In the ancient times, many drugs and diagnostic methods were introduced into China from abroad, for example, "frankincense, myrrh" was introduced into our country during the period of Zheng He's Sailing to the West Ocean and applied by TCM. Thus, in this modern society, the combination of both is also a trend.

Chinese medicine thinks the people as an organic whole, the body of the various parts of each other from the macro point of view, Chinese medicine has a macro accuracy, the lack of micro accuracy; and Western medicine is from the micro view of the problem, the use of analytical method, not the people as adults, but as a combination of various parts, there is a micro accuracy, but no macro accuracy. The theory tells us that the macro can command, including the microscopic; the microscopic can only explain and explain the macro, and not the commander and the macro. Therefore, traditional Chinese medicine can be assimilated by western medicine, western medicine cannot be assimilated by Chinese medicine. Specializes in traditional Chinese medicine combination therapy and individual therapy and medication is "uphold", is to mobilize the body's own forces to restore health, if necessary, will be specific for the disease itself. In Western medicine in addition to a small number of drugs need to individual treatment, combined with the main cause of the disease and symptoms, in order to remove the cause of the improvement of symptoms for the purpose.

\section{APPROACHES TO ACHIEVING THE COMBINATION}

\section{A. The Combination of TCM and Western Clinical Medicine}

Western medicine is good at the emergency treatment, surgery, and examinations, and its treatment depends on sophisticated equipment which is updated at high speed. Therefore, compared with the traditional "look, listening, questioning and pulse feeling", it has unparalleled advantages, especially in the treatment of "acute disease". However, the advantage of TCM lies in the health-keeping knowledge which is accumulated by thousands of years, such as work and rest, exercise, dietary therapy, breathing technique, eight trigrams boxing, herbal nourishment, 
martial arts, regulation of the mind and spirit, and healthpreserving. Its main feature lies in the disease prevention, which emphasizes the health concept to achieve longevity with high-quality life. Thus, its advantage is the chronic disease treatment.

The combination of TCM and western medicine has made great achievements: compound salvia pellet and Tongxinluo capsule based on the collateral disease theory have achieved good clinical efficacy and economic benefits. The application of tripterygium preparations in the area of Rheumatology is a big breakthrough in the RA treatment with the intervention of the combination of TCM and western medicine. The academician Chen Zhu proposed to use the arsenic trioxide injection to treat the relapse case of acute leukemia, and made great achievement. The efficacy of the compound realgar natural indigo tablets on the acute promyelocytic leukemia has been validated by the multi-center clinical trials, and achieved international recognition. ${ }^{[1]}$ The academician $\mathrm{Li}$ Leishi and his team drew on the experience of the immunosuppressive effects of tripterygium and first applied it to the nephritis. The project of "Applications of the Formula for Removing Stagnation and Clearing Heat in Type-2 Diabetes"of Tong Xiaolin and his research group placed the second in the National Science and Technology Progress Award ${ }^{[2]}$. The academician Zhang Boli, Wang Yongyan and their team conducted "the Research on the Treatment of Senile Vascular Dementia with tonifying kidney and resolving turbidi, which placed the second in the National Science and Technology Progress Award ${ }^{[3]}$.

The research of integrated traditional Chinese and Western medicine and standardization of clinical practice guidelines are important issues for the sustainable development of Chinese medicine industry. Health Organi-zation World (WHO) published the "international standard acupuncture point name" in 1991, which provided a standardized and standardized terms for International Acupuncture and moxibustion education. In recent years, the integrated Chinese and Western medicine research has been developed, and gradually move towards the standard. At present, the ISO / TC249 Secretariat have submitted 5 proposals for the international standard of Chinese medicine, and 2 of which have been approved by international standards. In 2007, China and South Korea, Japan and other countries and regions of the experts to complete the WHO "the traditional term for the international standards of the international standards". Now participate WHO international disease classification code (ICD-11), the drafting of traditional medical section. Chinese and Western medicine in many disciplines clinical research has achieved fruitful results in the deficiency and old medicine, skin diseases, health and rehabilitation medicine, psychosomatic medicine and other fields have been developed, such as "the study of TCM Syndromes of depression" to get the 2010 Annual National Science and technology progress award. These studies have not only made a useful exploration of the related Chinese and Western medicine research methodology, but also pay more attention to the limitations of previous studies and clinical disconnection.

\section{B. Experimental Study of the Combination of TCM and Western Medicine}

Currently TCM clinical research of the status quo is prospective study basic absence, many clinical studies lack of randomized controlled, poor repeatability in many therapies, prescription, reported the clinical curative effect of the reliability and science of suspicion. In the development of modern medicine in full swing today to remove the experience Medicine Hat, improvement of traditional Chinese medicine clinical research methodology must, change the past that clinical research based on case reports and experience summary, case summary based phenomenon. The introduction of evidence-based medicine, the use of clinical control and randomized controlled research methods, improve the reliability and scientific of the experimental results, to improve the scientific research, clinical level has a certain role in promoting. Hen Ziyin elaborated that kidney-yang deficiency is related with pituitary-adrenal-gonadal axis, and Chen Keji studied the blood-activating and stasisdissolving. The related theoretical researches have been used in clinical trial and gained outstanding clinical efficacy ${ }^{[4]}$. The academician Han Jisheng and his team studied the acupuncture aresthesia and gained great achievement: the mechanism of acupuncture analgesia spinal cord, acupuncture's treatment in shock, acusector at 36 in suppressing the adverse rising of stomach qi. The applications of Yin and Yang theory have penetrated into various areas of modern medicine, especially in elaborating the unity of opposites of gene regulation in organism and the restrictive relationship of dynamic equilibrium, and the corresponding achievements have been published in "Nature" and "Science".

Enhance the body's resistance; improve the sensitivity of chemotherapy patients. A lot of patients have experienced a long period of chemotherapy, in the long course of chemotherapy, the patient's physical constitution is constantly weakened, and the patient's resistance is also constantly emerging. I care group through close communication with doctors, make accurate diagnosis of patients, according to the type of syndrome, syndrome differentiation. To care with traditional Chinese medicine and therapeutic aspects of daily living. Chinese medicine stresses Peiyuan solid this, pay attention to nature and nurture, pay attention to filling in the empty, we according to the individual differences, for different patients be different diet and health education. Such as the spleen deficiency treated with Sijunzi decoction as the foundation to the spleen; Yin deficiency in Liuwei Dihuang Pill Based add and subtract with Tonifying Kidney Yin; lung yin deficiency with Tonga of Shashen Maidong reduction Yifei Yin etc.; at the same time with electric heating treatment and circulating immune therapy to improve the patient's immunity. After the transfer, the health of the patients has been greatly improved, which can better tolerate chemotherapy, to achieve the desired effect. Ease side effects.

Tumor patients in the course of chemotherapy, the majority will appear the following adverse reactions, such as physical weakness, immune function is low, bone marrow suppression, digestive disorders, inflammatory reaction, etc. In the application of Western medicine chemotherapy at the same time, we combine the 
traditional Chinese medicine acupoint injection at bilateral Zusanli alleviate nausea and vomiting, Acupuncture Sanyinjiao, Zusanli, Yanglingquan, Hegu acupoint improve immunity. During chemotherapy patients syndrome manifestations to the spleen and stomach deficiency, diet should be strengthening the spleen and stomach, such as barley lentil soup yam, Poria soup, lean meat, lotus seed, jujube porridge, radish, hawthorn and other, at the same time correct guide patients to use oral Decoction of Chinese medicine, traditional Chinese Medicine and traditional Chinese medicine traditional treatment methods, improve their condition. 3 through the psychological communication, reduce the psychological burden of patients, establish the confidence to overcome the disease. Everyone said that nine is the ten cancer death. That time, we need to do is to strengthen communication with patients, reduce the psychological burden, and establish the confidence to overcome the disease. When patients with depression, we communicate with patients, understanding the patient is not happy because of the psychological counseling. And improve the quality of life and prolong the survival time of patients. Many advanced cancer patients, after suffering from the disease, eats lying sleepless, shortness of breath, pain, the survival quality is quite low. We of the integrated Chinese and Western medicine combined with care, to diet and traditional Chinese medicine treatment, syndrome differentiation, using method of traditional Chinese medicine, traditional Chinese medicine and greatly improve the quality of life of patients and rekindle the survival of patients with lust.

\section{Using Modern Technology to Achieve the Modernization of TCM}

The key of the combination of TCM and western medicine lies in the former, including the study of Chinese herbal compound, single Chinese herb, effective fractions of TCM, and particular single component. The efficacy evaluation of compound medicine has become a basic study of the research and development of TCM and compounds. A research team led by Chen $\mathrm{Zhu}$, an academician Chinese Academy of Sciences, and a team led by Zheng Yong Qi from Yale University have become the highlight of pharmacological investigations in evaluating the efficacy of traditional Chinese medicine. Chen's research group illustrated the mechanism of Compound Realgar Natural Indigo Tablet in the treatment of leukemia from the molecular level and used it as an example to elaborate the prescription principle of different components of TCM. Prescriptions corresponding to syndromes contain the principle of prescriptions and syndromes, drugs and syndromes, amount and syndromes, combined prescriptions, and associated prescriptions. Both "Pharmacokinetic study of prescriptions and syndromes" and "Clinical and basic research of modern applications of classical prescriptions" won the second award of National Science and Technology Progress Award. The above achievements further prove that TCM is a great treasure and it is significant to achieve the modernization of TCM, ensure the quality and efficacy of drugs, and fully explore the mechanism of action of drugs, thus enabling more people to understand and use TCM.

Evidence based medicine is a scientific basis. The core idea is the determination of any medical decision-making, that is, the doctor to deal with the patient, the experts to develop treatment measures, the government to develop health policy and so on, should be based on the existing objective, the most reliable scientific basis. Along with the information boom, and because of the social and economic development, population aging, and the disease spectrum has changed. All medical personnel must be required to update the concept, to master the use of evidence-based medicine approach, continue to obtain new evidence, applied to clinical practice, and continuously improve the level of medical decision-making. In particular, the combination of Chinese and Western medicine is the cause of innovation, the development of medicine, in addition to the need to continue to obtain and correct application of evidence, but also the evidence of the provider. To study and sum up the achievements of Western medicine, collect and evaluate the existing literature, provide the best evidence, and make contribution to the promotion of Integrated Chinese and Western medicine to the world.

\section{WAYS TO PROMOTE THE INTERNATIONALIZATION OF THE COMBINATION OF TCM AND WESTERN MEDICINE}

The reason why TCM is not recognized by mainstream science is, in a large part, because it never goes beyond China's territory, enjoying its fame just in China. Therefore, TCM needs a big stage, the world stage, which is mainly achieved through the two ways discussed below.

\section{A. Establishing an International Multi-center Observation for Clinical Trials of TCM}

TCM has many drugs and therapies with noticeable effects, but because of the issues in the design of these clinical trials, it often fails to comply with the principles of RCT trials, thus making it difficult to achieve international recognition. Therefore, it is possible to conduct clinical trials in major medical institutions in different countries by improving the design. Although it is costly, it is able to achieve international recognition, and only by gaining recognition at home and abroad, can TCM enjoy high status and better combine with Western medicine.

Evidence based medicine, which is to follow the evidence of medicine, consider the clinical treatment of various diseases and decision-making, to the latest best and the most accurate evidence for the basis, and this design an evaluation of the level of evidence intensity, can evaluate the intensity of evidence of various clinical trials and clinical observation: there is more than a strong evidence for the design of large multi center, randomized, double blind, grouping controlled trials. This system is suitable for modern medicine, also applies to Chinese medicine, admit of no exception whatsoever. On the basis of RCT, we can perform a meta-analysis, or make a systematic it is a systematic and comprehensive collection of all the published clinical research articles, the screening of RCT for quantitative analysis, the most reliable conclusion. So we can greatly promote the academic progress in the field.

\section{B. Concentrating On the Quality of Papers and Publishing more SCI Papers}

Another important way to gain international recognition for TCM is to publish more SCI papers. 
Although reports about the uselessness of SCI papers are rampant, the trend of history provides a stimulus to the combination of Chinese and Western culture and medicine. A growing number of people overseas study traditional Chinese medicine and its theories. Currently, there are about 20 kinds of SCI journals integrated with medicine. ${ }^{[5]}$ Among them, Alternative Medicine Review has the highest impact factor, which was up to 4.857 in 2012, while the impact factor of Chinese Journal of Integrative Medicine increased from 0.79 in 2011 to 1.4 in 2013. The increase in the impact factor of Chinese medicine journals year by year indicates that papers of Chinese medicine are gaining international attention. Publishing high-quality papers enables more people to know about TCM and study it. The combination of Chinese and Western medicine is the combination of the traditional Chinese medicine knowledge and methods with the knowledge and methods of Western medicine and Western medicine, in order to improve the clinical efficacy, and to clarify the mechanism and a way to obtain new medical knowledge.

To sum up, the author believes that the combination of the two is not combining TCM with western medicine in china, but with that in foreign countries, which is a combination of thinking and methodology. Bringing foreign technology and medicine into use, western medicine in china is difficult to make a difference except with contempt for the traditional Chinese medicine and very few drugs or therapies with independent intellectual property rights. They just serve as the popularizer of Western pharmaceutical companies and help them sell drugs. Many bring back a large number of new drugs without clear efficacy from foreign countries to fool domestic patients and ask them to buy new drugs, which would cost them tens of thousands per month, but without prolonging the survival of them significantly. On the contrary, drug spending put a huge burden on the patient's family, throwing them into a serious economic crisis and causing numerous conflicts between doctors and patients ${ }^{[7]}$. Consequently, it is necessary to take full advantages of TCM and work out our own way to develop drugs and methods according to domestic situations, thus achieving more independent property rights and enhancing national self-confidence. By learning from Western technologies, we make great progress in TCM and the old saying "learn from the advanced technologies in the West in order to resist the invasion of the Western powers" still has significant meaning in the field of combining the TCM and Western medicine.

\section{REFERENCES}

[1] Zhu C. [Health care reform and development in China and healthy China strategy]. Zhonghua Yi Xue Za Zhi. vol.94,pp.20812085,2014

[2] Guo J, Chen H, Song J, Wang J, Zhao L, Tong X. Syndrome Differentiation of Diabetes by the Traditional Chinese Medicine according to Evidence-Based Medicine and Expert Consensus Opinion. Evid Based Complement Alternat Med. 2014. vol.2, pp 492193.

[3] Zhang J, Zhang B. Clinical research of traditional Chinese medicine in big data era. Front Med. vol.8, pp. 321-327.

[4] Wu T, Li Y, Zhen S, et al. Joint statement on promoting development of publication ethics among medical journals in China. J Evid Based Med. vol.4, pp. 214-216, 2011.

[5] Ernst E. Integrated medicine. Br J Gen Pract. vol.61, pp. 295, 2011 